\title{
A comparison between two different dried blood substrates in determination of psychoactive substances in postmortem samples
}

\author{
Matteo Moretti ${ }^{1}$ (1) - Alessandro Manfredi ${ }^{3}$. Francesca Freni ${ }^{1}$. Carlo Previderé ${ }^{1}$. Antonio Marco Maria Osculati ${ }^{1,2}$. \\ Pierangela Grignani ${ }^{1} \cdot$ Livio Tronconi $^{1,2}$. Claudia Carelli ${ }^{1}$. Claudia Vignali ${ }^{1} \cdot$ Luca Morini $^{1}$
}

Received: 14 July 2020 / Accepted: 20 November 2020 / Published online: 18 January 2021

(c) The Author(s) 2021

\begin{abstract}
Purpose Whatman ${ }^{\mathrm{TM}} 903$ cards represent a valid type of support for collection, storage, and analysis of dried blood spots (DBS). Whatman ${ }^{\mathrm{TM}}$ FTA (Flinders Technology Associates) are a type of cards soaked in chemicals that cause denaturation of proteins, while preserving DNA and ensuring the safe handling of DBS; to date, these cards are still rarely employed in forensic toxicology. The aim of this study was to analyze several psychoactive substances on not-dried blood on the two different cards and to compare the qualitative and quantitative results.

Methods Twenty cardiac postmortem blood samples were collected and deposed on Whatman ${ }^{\mathrm{TM}} 903$ and Whatman ${ }^{\mathrm{TM}}$ FTA cards. Spots and not-dried blood were analyzed following our validated and previously published liquid chromatographymass spectrometry methods.

Results We were able to identify: eight drugs of abuse and their metabolites ( 15 cases), five benzodiazepines and their metabolites ( 3 cases), six antidepressants ( 6 cases) and two antipsychotics ( 3 cases). We observed a perfect qualitative correspondence and a general good quantitative correlation between data obtained from not-dried blood and the two different DBS cards, except for alprazolam, diazepam, desmethyldiazepam, fluoxetine and sertraline, that showed a lower concentration on FTA. Additional experiments suggest that the chemicals, adsorbed on FTA, are not the cause of the loss of signal observed for the substances previously mentioned and that methanol should be preferred as extraction solvent.

Conclusions This study proved that FTA cards are a good and a hazard-free alternative sample storage method for analysis of several psychoactive substances in postmortem blood.
\end{abstract}

Keywords Flinders Technology Associates card · Dried blood spots · Postmortem · Psychoactive substances

Supplementary Information The online version contains supplementary material available at https://doi.org/10.1007/s1141 9-020-00567-2.

Matteo Moretti

matteo.moretti01@universitadipavia.it

1 Department of Public Health, Experimental and Forensic Medicine, University of Pavia, via Forlanini 12, Pavia, Italy

2 Legal Medicine Operative Unit, IRCCS Fondazione Mondino, Pavia, Via Mondino 2, 27100 Pavia, PV, Italy

3 Department of Medicine, Surgery and Health, University of Trieste, Piazzale Europa 1, Trieste, Italy

\section{Introduction}

Dried blood spot (DBS) is a sampling technique that involves the application of very small volumes of blood on a paperbased substrate, which is eventually left to dry; the spots can then subsequently be easily extracted and analyzed. To date, a wide variety of substances has been tested on dried blood samples. The first description of this technique dates back to 1963 by Guthrie et al., who utilized the spots in neonatal screening for congenital phenylketonuria diagnosis [1]. Thanks to its compatibility with a large number of bioanalytical procedures, such as chromatography, mass spectrometry, DNA analysis and enzyme immunoassays, over the years these samples have been studied in several research fields [2]. Among these, the most important were preclinical development of drugs [3], clinical pharmacology [4], therapeutic drug monitoring [5-7], control of environmental 
contaminants [8], surveillance for microbiological diseases [9], genetics [10] and, more recently, forensic toxicology [11].

Numerous methods, particularly dedicated to the identification and quantification of psychotropic molecules and substances of abuse, have been developed and validated on DBS. Most of these studies focused on Whatman ${ }^{\mathrm{TM}} 903$ cards. This paper-based substrate allows to collect a certain volume of blood (about $75-80 \mu \mathrm{L}$ per spot) on a fixed surface area (1.27 cm diameter). A good qualitative and quantitative correlation was observed for drugs of abuse, benzodiazepines, antidepressants and antipsychotics measured in fresh blood and in samples dried on Whatman ${ }^{\mathrm{TM}}$ 903 cards, collected from postmortem samples. In particular, the analysis of the whole blood spot, rather than of a fixed portion, may limit the variability due to the hematocrit, as well as the different coefficient of diffusion of the monitored substances on the paper substrate [12-14].

The Whatman ${ }^{\mathrm{TM}}$ FTA (Flinders Technology Associates) cards represent another valid type of support for the collection, transport, storage and analysis of biological samples. One of the main application areas is genetics [15], as these paper supports are soaked in chemicals that cause lysis of cells, denaturation of proteins and protect nucleic acids from nuclease action, oxidation and UV damage, keeping them intact for future analysis. The papers also rapidly inactivate any virus or organism present in the sample and prevent the growth of microorganisms, ensuring the safe handling of cards without risk of biohazards. This aspect is particularly important nowadays, considering the current emergency linked to the COVID-19 outbreak.

To date, the use of FTA cards in forensic toxicology has been limited and tested on a few classes of psychotropic substances, such as benzodiazepines. The authors spiked 26 analytes among benzodiazepines, z-drugs and metabolites on FTA-Drug Metabolism and Pharmacokinetics (DMPK) cards and observed a high quantitative reliability of the method [16]. Barfield and coauthors developed a robust and sensitive method for the determination of paroxetine in dried plasma spots, using FTA substrate. They concluded that FTA cards can be a reliable alternative matrix to store plasma and whole blood for therapeutic drug monitoring of medicines [17]. However, these two studies are only based on spiked samples, and have not been applied to real positive blood or plasma samples. A comparative study between five different DBS cards for the determination of six immunosuppressants has been performed by Koster and coauthors. The authors observed different performance results depending on the concentration of the analyte investigated [18].

The objective of this study was to compare quantitative and qualitative results obtained from analysis of not-dried postmortem blood samples, and those for blood depositions using two different paper substrates.

\section{Materials and methods}

\section{Chemicals}

Diazepam, desmethyldiazepam, chlordesmethyldiazepam, alprazolam, clonazepam, 7-aminoclonazepam, bromazepam, flurazepam, desalkylflurazepam, midazolam, triazolam, zolpidem, clotiapine, amitriptyline, nortriptyline, haloperidol, fluoxetine, nortriptyline, fluvoxamine, promazine, chlorpromazine, aripiprazole, mirtazapine, desmethylmirtazapine, maprotiline, venlafaxine, desvenlafaxine, sertraline, paliperidone, citalopram, desmethylcitalopram, diazepam-D5, 7-aminoclonazepam-D4 alprazolam-D5, quetiapine-D8, clozapine-D4, and citalopram-D4 methanolic solutions were obtained from Sigma-Aldrich (Milan, Italy); cocaine, cocaethylene (CE), ecgonine methylester (EME), benzoylecgonine (BE), methadone, 2-ethylidene1,5-dimethyl-3,3-diphenylpyrrolidine (EDDP), morphine, codeine, ketamine, norketamine, amphetamine, methamphetamine, 3,4-methylenedioxymethamphetamine (MDMA), 3,4-methylenedioxy- $N$-ethylamphetamine (MDEA), 1,3-benzodioxolyl- $N$-methylbutanamine (MBDB), 3,4-methylenedioxyamphetamine (MDA), $\alpha$-pyrrolidinohexiophenone ( $\alpha$-PHP), $\alpha$-pyrrolidinopentiophenone ( $\alpha$-PVP), cocaine-D3, morphine-D3, methadone-D3, EME-D3, ketamine-D4, MDMA-D4, mephedrone-D5, and methanolic solutions were obtained from Cerilliant (Milan, Italy). Liquid chromatography-mass spectrometry (LC-MS) grade methanol, acetonitrile, formic acid, dichloromethane, 2-propanol and ammonia were purchased from Carlo Erba S.R.L. (Milan, Italy).

The mobile phase consisted of an aqueous solution with $0.1 \%(\mathrm{v} / \mathrm{v})$ formic acid (A) and acetonitrile with $0.1 \%(\mathrm{v} / \mathrm{v})$ formic acid (B).

\section{Instrumentation}

Liquid chromatographic tandem mass spectrometric (LC-MS/MS) analyses were performed on an Agilent 1100-1200 Series system (Agilent Technologies, Palo Alto, CA, USA) coupled with a 4000 Q-TRAP (AB SCIEX, Foster City, CA, USA). The LC instrumentation composed of a binary pump, an isocratic pump and an autosampler maintained at room temperature during analysis. The injector needle was externally washed with methanol ( $3 \mathrm{~s})$ prior to any injection. A Kinetex $\mathrm{C} 18 \mathrm{col}-$ umn $(100 \times 2.1 \mathrm{~mm}$ i.d., $2.6 \mu \mathrm{m}$ particle size) (Phenomenex, Castelmaggiore, BO, Italy) was kept at $35^{\circ} \mathrm{C}$ during the analysis. The chromatographic separation was carried out in reverse phase, with mobile phases consisting of $0.1 \%(\mathrm{v} / \mathrm{v})$ formic acid in bidistilled water and $0.1 \%(\mathrm{v} / \mathrm{v})$ 
formic acid in acetonitrile. The elution was performed in gradient mode and three different elutions were used and were previously published [13-15]. Multiple reaction monitoring (MRM) was optimized using nitrogen as collision gas (with pressure set at level 5) and a dwell time of 30-50 ms. Two transitions for each substance were chosen for identification. To guarantee the best sensitivity, the MRM transitions were divided into two 4 groups. Data acquisition and elaboration were performed by the Analyst ${ }^{\circledR}$ software (version 1.5.1, AB SCIEX).

\section{Sample analysis protocol}

Twenty cardiac blood samples, collected from 20 different autopsy cases which tested positive for at least one psychoactive substance during routine toxicological analyses, were included in the study. Exclusion criteria were: presence of blood clots in the plastic tube (sample not homogeneous); low blood amount $(<10 \mathrm{~mL})$; blood collected from putrefied corpses; and addition of preservatives. After the collection, blood samples were stored in plastic tube at $-20{ }^{\circ} \mathrm{C}$ until the deposition on the DBS cards. Aliquots of $85 \mu \mathrm{L}$ of blood were pipetted on the filter cards (three different spots on Whatman ${ }^{\mathrm{TM}}$ 903TM and three different spots on Whatman ${ }^{\mathrm{TM}}$ FTA, Sigma-Aldrich, Milan, Italy) and left to dry (for about $2 \mathrm{~h}$ ), keeping them in the dark, at room temperature. Blood spots were analyzed within $24 \mathrm{~h}$ from the deposition.

For each spot, the whole blood stain (a disk of about $13 \mathrm{~mm}$ diameter) was cut and put into a glass tube, containing 1-mL phosphate buffer solution at pH 6 and all the deuterated internal standards cited above at the concentration of $100 \mathrm{ng} / \mathrm{mL}$. The solutions were sonicated for $10 \mathrm{~min}$, vortexed for $10 \mathrm{~s}$ and finally centrifuged at $4000 \mathrm{~g}$ for $5 \mathrm{~min}$. Supernatant solutions were separated from the filter cards and purified through a Bond Elut Certify I solid-phase extraction (SPE, $200 \mathrm{mg}$ ) cartridge (CPS Analitica, Milan, Italy). The cartridges were initially activated with $2-\mathrm{mL}$ methanol, then rinsed with 2-mL phosphate buffer solution at $\mathrm{pH} 6$, before loading samples solutions. The columns were then washed with 2-mL deionized water, $3 \mathrm{~mL} 0.1 \mathrm{M} \mathrm{HCl}$ and finally $5-\mathrm{mL}$ methanol. The analytes elution was carried out with 2-mL dichloromethane-isopropanol mixture $(8: 2$ $\mathrm{v} / \mathrm{v})$ with $2 \%$ ammonia solution. The eluate solution was dried under nitrogen stream, and reconstituted in $200-\mu \mathrm{L}$ mobile phase; finally, $5 \mu \mathrm{L}$ was injected in the LC-MS/ MS system. The same procedure was applied also to three aliquots, of $85 \mu \mathrm{L}$ each, of not-dried blood.

The method for all the analytical procedures has already been validated and previously published [12-14].

\section{Evaluation of matrix effects, recovery, extraction efficiency and batch stability}

Methanolic working solutions containing 45 psychoactive substances (solution A) and internal standards (solution B) were freshly prepared at the concentration of $1000 \mathrm{ng} / \mathrm{mL}$. Twenty microliters of solution A and $20 \mu \mathrm{L}$ of solution B were added to $1980 \mu \mathrm{L}$ blank blood and to $1980 \mu \mathrm{L}$ phosphate buffer solution. Fifty microliters of spiked blood sample were deposed on five FTA cards. The same procedure was applied to spiked buffer solution. The cards were let to dry and, eventually, processed as described in the previous paragraph. Fifty microliters of blank blood samples and blank phosphate buffer solutions were deposed to FTA cards and processed using the same procedure. The solutions $\mathrm{A}$ and B were spiked to blank samples after the SPE extraction. Phosphate buffer solutions and blood samples were also analyzed without deposition on FTA cards. All the analyses were carried out in quintuplicate. Batch stability was measured by injecting the same spiked samples at the beginning and at the end of the sequence (about $24 \mathrm{~h}$ ).

\section{Correlation between 903 and FTA cards in spiked samples without biological matrix}

Due to controversial data obtained for some molecules detected in real samples (issue discussed further below), another experiment was set up during the study. Forty-five psychoactive substances and internal standards were added to a phosphate buffer solution at a final concentration of $1000 \mathrm{ng} / \mathrm{mL}$. Fifty microliters were deposed on FTA and 903 cards and left to dry. The phosphate buffer solution and the cards were then processed, following the sample treatment procedure described above. Twelve different replicates were performed.

\section{Results}

Twenty-one different substances were detected, at least once, among the 20 real blood samples. The identified substances were: cocaine $(n=11), \mathrm{BE}(n=11)$, EME $(n=11)$, CE $(n=3)$, morphine $(n=4)$, codeine $(n=4)$, methadone $(n=3)$, EDDP $(n=3), 7$-amino-clonazepam $(n=2)$, citalopram $(n=2)$, diazepam $(n=2)$, quetiapine $(n=2)$, venlafaxine $(n=2)$, desvenlafaxine $(n=2)$, alprazolam, amitriptyline, bromazepam, desmethyldiazepam, fluoxetine, haloperidol and sertraline. A total of 69 measurements were performed. All the molecules were detected in not-dried blood, in blood dried on classic 903 DBS and in blood dried on FTA cards. The concentrations and the standard deviations are shown in Table 1. Least-squares regression analyses demonstrated a good quantitative correlation between data 
Table 1 Measured concentrations in not-dried blood, Whatman ${ }^{\mathrm{TM}} 903$ and Whatman ${ }^{\mathrm{TM}}$ FTA

\begin{tabular}{|c|c|c|c|c|c|c|}
\hline \multirow[t]{2}{*}{ Substance } & \multicolumn{2}{|c|}{$\begin{array}{l}\text { Conc. in not-dried } \\
\text { blood }(\mathrm{ng} / \mathrm{mL})\end{array}$} & \multicolumn{2}{|c|}{$\begin{array}{l}\text { Conc. in Whatman } \\
903 \text { DBS }(\mathrm{ng} / \mathrm{mL})\end{array}$} & \multicolumn{2}{|c|}{$\begin{array}{l}\text { Conc. in Whatman }{ }^{\mathrm{TM}} \\
\text { FTA DBS }(\mathrm{ng} / \mathrm{mL})\end{array}$} \\
\hline & Mean & SD & Mean & SD & Mean & SD \\
\hline \multirow[t]{11}{*}{ Cocaine $(n=11)$} & 56.7 & 2.6 & 63.5 & 3.3 & 62.6 & 1.8 \\
\hline & 302 & 25.1 & 295 & 12.5 & 295 & 10.4 \\
\hline & 155 & 11.6 & 181 & 24.9 & 197 & 34.2 \\
\hline & 679 & 30.7 & 686 & 163 & 670 & 76.2 \\
\hline & 54.7 & 8.8 & 46.6 & 9.6 & 73.6 & 11.3 \\
\hline & 52.7 & 5.3 & 50.3 & 6.4 & 57.3 & 9.2 \\
\hline & 40 & 1.4 & 50.5 & 6.7 & 45.7 & 4 \\
\hline & 231 & 16.4 & 231 & 4 & 244 & 13.1 \\
\hline & 148 & 13.7 & 136 & 13.5 & 158 & 13.3 \\
\hline & 1350 & 40 & 1340 & 65.6 & 1357 & 92.9 \\
\hline & 85.3 & 3.1 & 71 & 5.8 & 70.1 & 19.6 \\
\hline \multirow[t]{11}{*}{$\mathrm{BE}(n=11)$} & 443 & 32.6 & 520 & 37.3 & 517 & 12.3 \\
\hline & 1170 & 45.1 & 1060 & 104 & 1180 & 81.9 \\
\hline & 745 & 23.1 & 718 & 27.6 & 873 & 13.8 \\
\hline & 2140 & 217 & 1900 & 28.6 & 2830 & 705 \\
\hline & 1150 & 1230 & 1230 & 20.8 & 1280 & 145 \\
\hline & 441 & 27.9 & 541 & 17.9 & 526 & 15.3 \\
\hline & 87.1 & 14 & 117 & 16.8 & 105 & 5.8 \\
\hline & 771 & 27.8 & 709 & 13.9 & 792 & 17.6 \\
\hline & 948 & 49.2 & 1010 & 108 & 943 & 49.8 \\
\hline & 4170 & 270 & 3600 & 130 & 3750 & 261 \\
\hline & 3700 & 431 & 3590 & 431 & 3750 & 329 \\
\hline \multirow[t]{11}{*}{$\operatorname{EME}(n=11)$} & 192 & 6.4 & 178 & 25.5 & 226 & 24.9 \\
\hline & 608 & 7.8 & 543 & 56 & 564 & 23.8 \\
\hline & 381 & 21.2 & 364 & 28.5 & 427 & 69.9 \\
\hline & 2150 & 70 & 2130 & 172 & 2180 & 197 \\
\hline & 476 & 22 & 403 & 61.8 & 556 & 55.3 \\
\hline & 108 & 3.6 & 108 & 9 & 148 & 8.4 \\
\hline & 52 & 3.8 & 59.6 & 8.7 & 64.4 & 3.7 \\
\hline & 439 & 10.5 & 477 & 18.2 & 456 & 47.9 \\
\hline & 335 & 7.8 & 307 & 6.6 & 335 & 8.6 \\
\hline & 3120 & 138 & 3310 & 221 & 3410 & 85 \\
\hline & 846 & 30 & 894 & 40.3 & 952 & 37.7 \\
\hline \multirow[t]{3}{*}{$\mathrm{CE}(n=3)$} & 103 & 4.3 & 102 & 12.6 & 107 & 6.4 \\
\hline & 104 & 11.5 & 96.6 & 10.2 & 108 & 12.7 \\
\hline & 81.1 & 5.2 & 76 & 4.4 & 70.7 & 6.3 \\
\hline \multirow[t]{4}{*}{ Morphine $(n=4)$} & 34.4 & 3.3 & 34.6 & 0.7 & 33.6 & 3.4 \\
\hline & 206 & 7.5 & 240 & 18 & 208 & 32 \\
\hline & 155 & 4.6 & 98.9 & 8.1 & 149 & 29.1 \\
\hline & 237 & 11.5 & 267 & 41.6 & 299 & 23.1 \\
\hline \multirow[t]{4}{*}{ Codeine $(n=4)$} & 19.2 & 1.2 & 19.3 & 1.5 & 20.4 & 1.3 \\
\hline & 27.5 & 0.8 & 31.8 & 2.3 & 31.9 & 1.3 \\
\hline & 21.8 & 1.5 & 22.2 & 1.1 & 27.3 & 6.6 \\
\hline & 31.9 & 0.4 & 34.6 & 2.4 & 31.5 & 3.1 \\
\hline \multirow[t]{3}{*}{ Methadone $(n=3)$} & 781 & 231 & 781 & 91.1 & 527 & 147 \\
\hline & 1870 & 1960 & 785 & 33 & 499 & 12.5 \\
\hline & 241 & 13 & 264 & 43.1 & 162 & 23.2 \\
\hline
\end{tabular}


Table 1 (continued)

\begin{tabular}{|c|c|c|c|c|c|c|}
\hline \multirow[t]{2}{*}{ Substance } & \multicolumn{2}{|c|}{$\begin{array}{l}\text { Conc. in not-dried } \\
\text { blood (ng/mL) }\end{array}$} & \multicolumn{2}{|c|}{$\begin{array}{l}\text { Conc. in Whatman }{ }^{\mathrm{TM}} \\
903 \text { DBS (ng/mL) }\end{array}$} & \multicolumn{2}{|c|}{$\begin{array}{l}\text { Conc. in Whatman }{ }^{\mathrm{TM}} \\
\text { FTA DBS (ng/mL) }\end{array}$} \\
\hline & Mean & SD & Mean & SD & Mean & SD \\
\hline \multirow[t]{3}{*}{$\operatorname{EDDP}(n=3)$} & 102 & 13.2 & 56.9 & 6.2 & 50.7 & 13.6 \\
\hline & 158 & 169 & 60.2 & 2.5 & 49.4 & 2.5 \\
\hline & 35.9 & 1.6 & 45.2 & 9.3 & 38.6 & 2.8 \\
\hline \multirow[t]{2}{*}{ Quetiapine $(n=2)$} & 276 & 30.5 & 223 & 16.5 & 268 & 44 \\
\hline & 6700 & 1060 & 5930 & 621 & 8730 & 1710 \\
\hline \multirow[t]{2}{*}{ Citalopram $(n=2)$} & 11.1 & 0.2 & 9.8 & 1.5 & 6.5 & 0.9 \\
\hline & 345 & 34.7 & 359 & 14.5 & 295 & 24 \\
\hline \multirow[t]{2}{*}{ Venlafaxine $(n=2)$} & 1810 & 233 & 2120 & 537 & 2070 & 548 \\
\hline & 2590 & 50.3 & 2490 & 17.7 & 2480 & 444 \\
\hline \multirow[t]{2}{*}{ Desvenlafaxine $(n=2)$} & 541 & 82.1 & 486 & 15 & 545 & 25.8 \\
\hline & 832 & 73.4 & 976 & 329 & 819 & 82 \\
\hline \multirow[t]{2}{*}{ Diazepam $(n=2)$} & 18.5 & 2 & 19.3 & 2.4 & 6.3 & 1.1 \\
\hline & 58.1 & 3.6 & 43.7 & 5.3 & 23.6 & 5.2 \\
\hline \multirow[t]{2}{*}{ 7-Amino-clonazepam $(n=2)$} & 33.9 & 4.4 & 29.8 & 3.3 & 41.4 & 2.5 \\
\hline & 82.7 & 4.5 & 72.5 & 1.4 & 85.2 & 12.2 \\
\hline Alprazolam $(n=1)$ & 37.8 & 2.9 & 34.6 & 4.9 & 18.9 & 2.5 \\
\hline Amitriptyline $(n=1)$ & 66.7 & 10.9 & 56.3 & 4.3 & 42.1 & 9.7 \\
\hline Bromazepam $(n=1)$ & 247 & 15.7 & 220 & 24.1 & 217 & 15.6 \\
\hline Desmethyldiazepam $(n=1)$ & 157 & 5.7 & 145 & 15.3 & 44.4 & 6.9 \\
\hline Fluoxetine $(n=1)$ & 1230 & 94.5 & 1310 & 95.4 & 185 & 67 \\
\hline Haloperidol $(n=1)$ & 7 & 0.2 & 7.9 & 0.8 & 6.5 & 0.9 \\
\hline Sertraline $(n=1)$ & 530 & 106 & 395 & 31.6 & 28.1 & 2 \\
\hline
\end{tabular}

obtained from not-dried blood and the two different DBS cards, with $r^{2}=0.9782$ and 0.9405 for 903 and FTA, respectively $(\rho<0.005)$. The Spearman's coefficient $r_{\mathrm{s}}$ also confirmed the good correlation, with a calculated value between not-dried blood and 903 DBS of $0.8922(\rho<0.0001)$ and between not-dried blood and FTA of $0.8898(\rho<0.0001)$. A Bland-Altman plot was set up for the results obtained from the two cards, in comparison to the data measured in not-dried blood. Plots are reported in Figs. 1 and 2. A general good agreement was observed between not-dried blood and the two dried alternatives. Concentrations of fluoxetine and sertraline, measured in FTA0 DBS, were about tenfold lower than those measured in not-dried blood and significantly lower than the ones observed in 903 DBS. A lower concentration in FTA DBS was also observed for some benzodiazepines, namely alprazolam, diazepam and desmethyldiazepam.

Matrix effects, recovery and extraction efficiency were considered acceptable for all the substances, except for amitriptyline, venlafaxine, desvenlafaxine and codeine. All the results are reported as supplementary material (Table S1). Extraction efficiency was measured by comparing the signals obtained from spiked blood samples deposed on FTA cards and spiked bloods samples directly processed. Recovery was
Fig. 1 Bland-Altman plot for the results obtained from Whatman $^{\mathrm{TM}} 903$ card, in comparison to the data measured in notdried blood

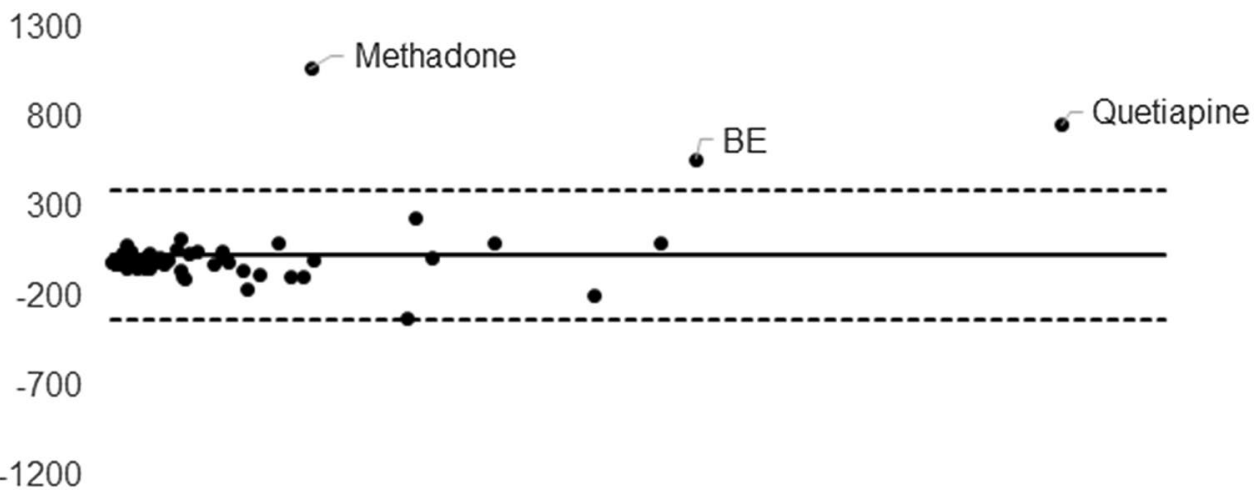


Fig. 2 Bland-Altman plot for the results obtained from Whatman ${ }^{\mathrm{TM}}$ FTA cards, in comparison to the data measured in not-dried blood

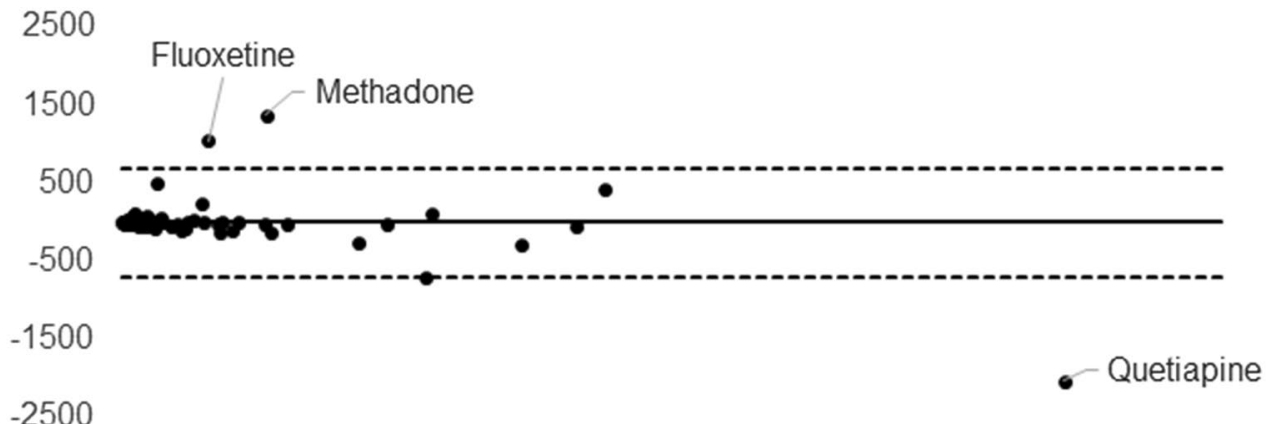

reported as the percentage of standard solutions spiked on DBS cards before SPE extraction with same samples spiked at the same concentration after SPE procedure.

In particular, matrix effects were considered negligible for almost all the substances, except for amphetamine, methamphetamine, MDMA, MBDB and chlorpromazine, for which a slight ion suppression was observed. A general high recovery percentage was observed for most of the drugs of abuse. On the contrary, low recoveries were measured for most of benzodiazepines. The lower $\mathrm{p} K_{\mathrm{a}}$ of these molecules, together with their chemical characteristics represent the main reason for a low recovery. However, the SPE procedure guarantees a good repeatability for those substances, as already demonstrated in a previous paper [13]. All the monitored molecules were stable within the sequence run (about $24 \mathrm{~h}$ ).

To evaluate a potential degradation of these molecules after deposition on the FTA (e.g., due to chemicals adsorbed in the card), a further experiment was performed. Fifty microliters phosphate buffer solution containing all the 21 substances detected in real samples, together with other 24 psychoactive substances with different physical and chemical characteristics, were deposed on the two different DBS cards ( $n=12$ replicates for each), left to dry, processed as described above, and matched to data obtained from the analyses of a same volume of not-dried phosphate buffer solution. The peak areas of each substance, obtained from all the 903 and FTA samples, after normalization by proper internal standards, were compared with those ones obtained from the standard solutions. The 903/buffer solution and FTA/ buffer solution ratios are reported in Figs. 3 and 4, respectively. The peak area ratios were generally in better agreement between FTA DBS and phosphate buffer solution rather than those measured in samples deposed on 903 DBS. Yet, the massive loss of signal measured for fluoxetine and sertraline in real dried blood samples, was not observed

300

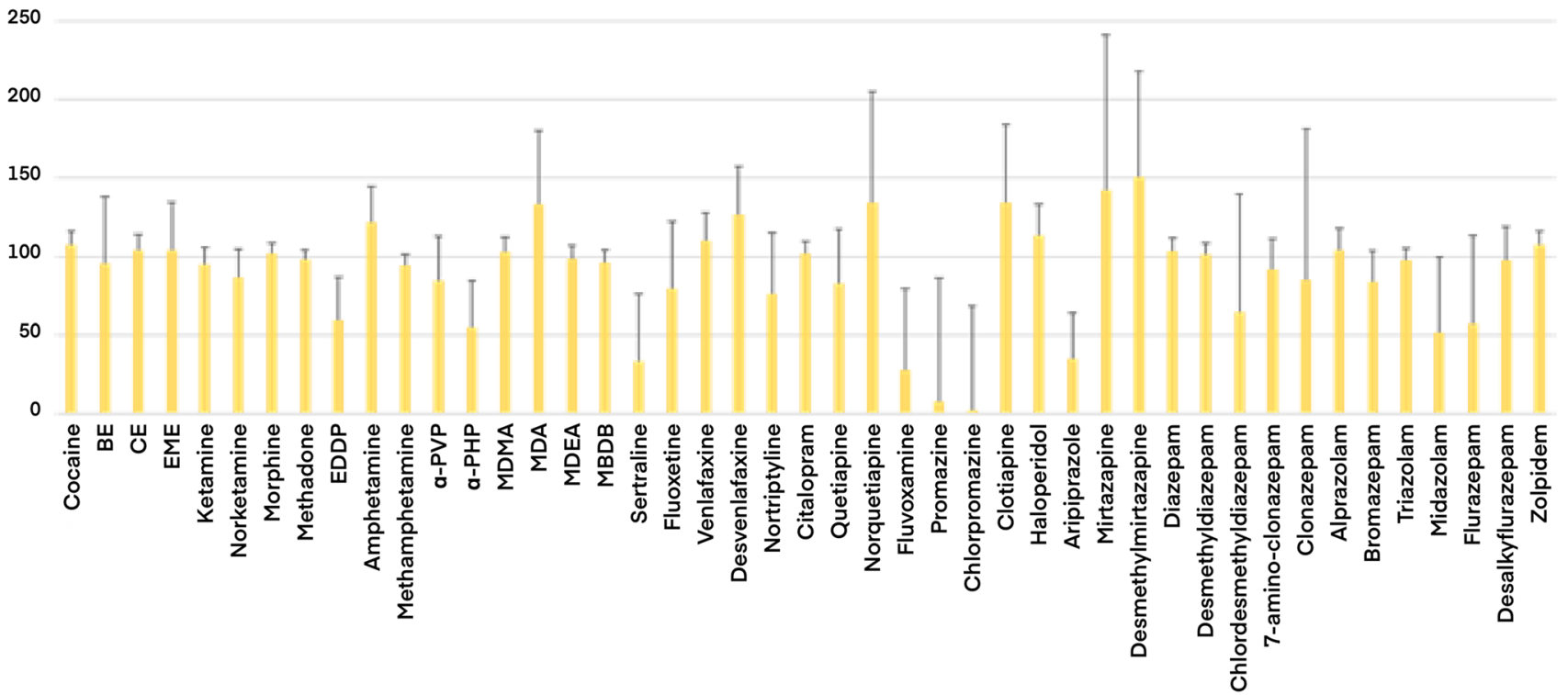

Fig. 3 903/buffer solution peak area ratios at fixed concentration (100 ng/mL) 


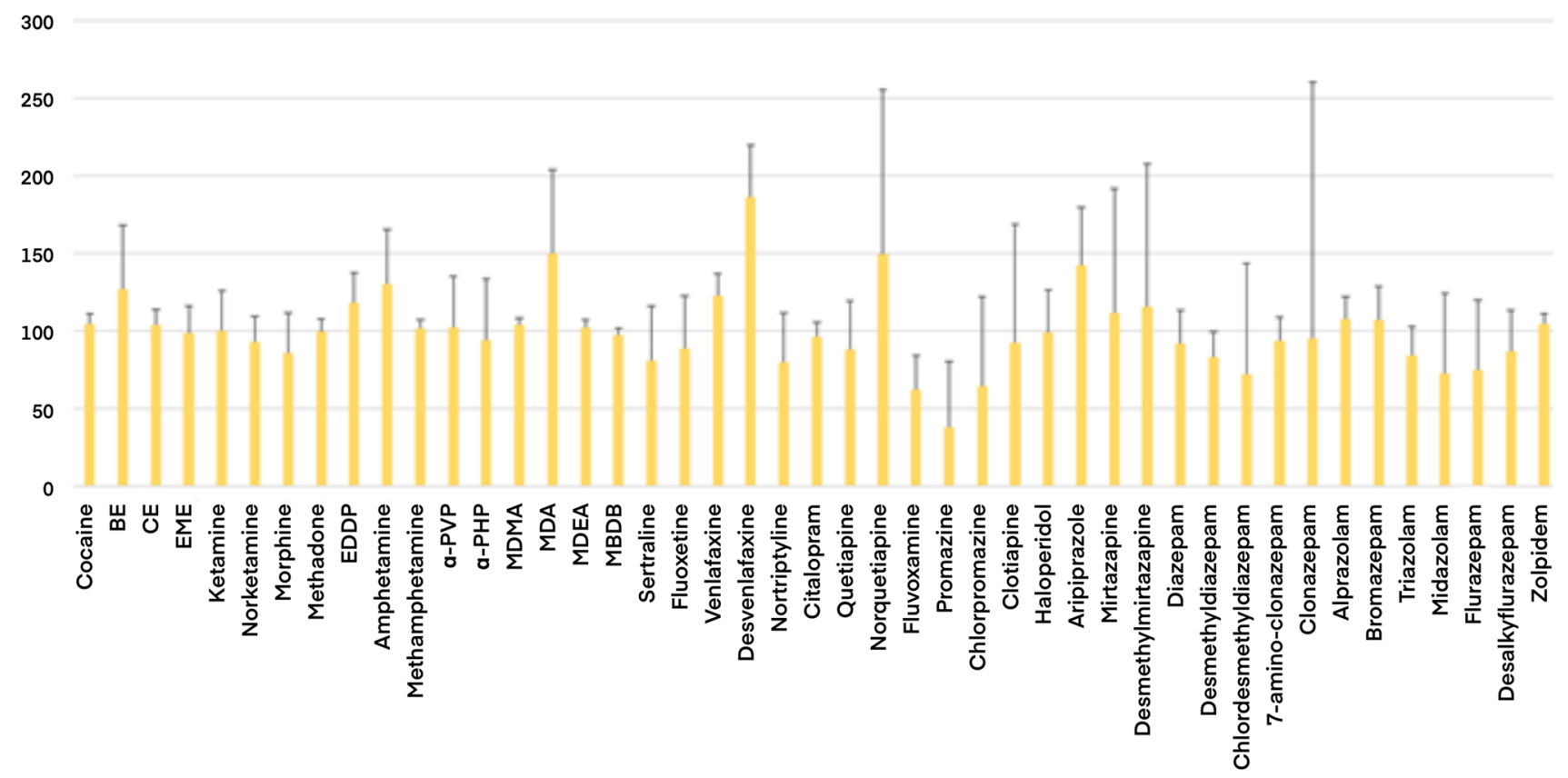

Fig. 4 FTA/buffer solution peak area ratios at fixed concentration $(100 \mathrm{ng} / \mathrm{mL})$

in spiked phosphate buffer solution dried on the same FTA cards.

\section{Discussion}

This study was performed to compare the qualitative and quantitative results obtained from blood stored under routine conditions, and blood dried on two different DBS cards. In particular, previous studies on postmortem samples, performed on Whatman ${ }^{\mathrm{TM}} 903$ substrates, demonstrated that the data were in good agreement with the ones carried out in not-dried blood [12-14]. However, FTA cards differ from 903 ones because of the chemicals adsorbed on the paper substrate, that allow to lyse cells and denature proteins, while protecting nucleic acids from nuclease action, UV, and oxidative damage. This factor may influence the stability of some molecules of forensic interest, such as psychoactive substances.

Preliminary data, obtained from 20 positive postmortem samples, confirmed that 903 cards provide similar results in terms of identification and quantification of the monitored substances. In particular, $<10 \%$ of the data obtained from the 903 DBS yielded a concentration lower than $85 \%$ respect to that measured in blood stored at routine conditions. A similar good agreement was also assessed by the response of the Bland-Altman plot and the Spearman's rho value. Moreover, a general good correlation was observed between data, carried out through the analyses of blood samples, deposed on FTA cards, and analyses of not-dried blood. Cocaine and its main metabolites were the most frequently detected substances. Eleven out of 20 cases were positive for cocaine, BE and EME. The comparison between concentrations measured in stored blood and samples dried on cards confirmed previously published data, and proved that DBS are a good alternative sample storage, independently on the paper substrate type. Similar results were obtained for morphine and codeine. On the contrary, one case for methadone and two cases for 2-ethylidene-1,5-dimethyl-3,3-diphenylpyrrolidine (EDDP) provided lower concentrations in cards compared to the ones measured in not-dried blood. Most of the antidepressants yielded similar quantitative results in routinely stored blood and in blood dried on the two different cards. However, an almost complete degradation was reported for two antidepressants, namely sertraline and fluoxetine. Moreover, also results obtained from the quantitative determination of alprazolam, diazepam and desmethyldiazepam in blood samples, dried on FTA substrate, were significantly lower than those measured in blood stored at routine conditions. Initially, it was hypothesized that chemicals adsorbed on the FTA could have influenced the stability of these compounds: a further experiment, without biological matrix, was, therefore, set up, to assess this hypothesis. Forty-five psychoactive substances, including all those ones detected in real samples, were diluted in a phosphate buffer solution at a final concentration of $1000 \mathrm{ng} / \mathrm{mL}$. Eventually, $50 \mu \mathrm{L}$ of the solution was deposed on 12 different spots of FTA and 12 spots of 903 filter papers. The 12 samples were measured and compared to 12 samples of buffer solution at the same volume and concentration. The results from this experiment, 
reported in Figs. 3 and 4, were clearly in contrast with those obtained from real postmortem samples. Indeed, a good correlation between peak area ratios, measured in solution, and in samples deposed on FTA substrate, was observed for most of the compounds, and was generally higher than the correlation between not-dried buffer solution and samples dried on 903 cards. Moreover, the standard deviations, measured for FTA samples, were lower than the ones calculated for samples deposed on 903 papers. The experiments on standard solutions highlighted a degradation of phenothiazines on DBS cards, independently on the paper substrate. In fact, an almost complete loss of promazine was observed in 903 card, together with a significant decrease of the signal of chlorpromazine. A more limited loss was achieved using the FTA cards. However, preliminary data on standard solutions suggested that the quantitative determination of phenothiazines in samples deposed in DBS should be interpreted with caution.

The results of the experiment with phosphate standard solutions confirmed that chemicals adsorbed on the FTA card are not the cause of the loss of signal observed for sertraline, fluoxetine and the three benzodiazepines. Therefore, we supposed that the differences between data obtained from real samples and standard solutions concern the biological matrix. We deposed other aliquots of blood samples, positive for sertraline, fluoxetine and diazepam (three aliquots for each sample) on FTA and 903 cards. The extraction was performed by adding $1 \mathrm{~mL}$ methanol, instead of $1 \mathrm{~mL}$ phosphate buffer. Two microliters of buffer solution were added to the samples just before SPE procedure. The rest of sample preparation was the same as the one described above. The analytical data were compared to the ones obtained from blood samples stored at routine conditions, and extracted with $1 \mathrm{~mL}$ methanol. The differences between peak area ratios measured in blood samples dried on FTA and extracted with methanol were not significantly different ( $<15 \%$ standard deviations) from the ones measured in blood samples deposed on 903 cards and in not-dried blood samples. These results suggested that methanol should be preferred as extraction solvent, when FTA cards are used.

\section{Conclusion}

This study proved that FTA DBS cards are a good and a hazard-free alternative sample storage for analysis of most of the psychoactive substances detected in postmortem blood. Preliminary data, obtained from 20 real cases, confirmed a good qualitative and quantitative agreement between concentrations of different drugs of abuse, antidepressants, antipsychotics, benzodiazepines and metabolites, measured in blood stored at routine conditions, and blood dried on DBS cards, independently on the type of paper substrate, except for diazepam, desmethyldiazepam, alprazolam, fluoxetine and sertraline, that provided a lower extraction from FTA cards. Phosphate buffer solution did not provide a high extraction efficiency, when applied to FTA cards, for all the substances detected. Methanol seemed to increase the extraction performance, and therefore, should be preferred whenever FTA cards are used.

Author contributions All the authors contributed to the study conception and design. Material preparation, sample collection and data collection were performed by Luca Morini, Matteo Moretti, Alessandro Manfredi, Francesca Freni, Carlo Previderè, and Antonio Marco Maria Osculati. Analysis were performed by Francesca Freni, Claudia Vignali, Luca Morini, and Pierangela Grignani. The first draft of the manuscript was written by Matteo Moretti, Alessandro Manfredi and Francesca Freni. Luca Morini, Claudia Carelli, Claudia Vignali, Livio Tronconi, and Antonio Marco Maria Osculati supervised, reviewed and edited the manuscript. All the authors read and approved the final manuscript.

Funding Open Access funding provided by Università degli Studi di Pavia.

\section{Compliance with ethical standards}

Conflict of interest The authors declare that they have no conflict of interest.

Ethical approval In accordance with Italian Law, this research was performed on small portions of biological samples routinely taken during autopsies that were already examined for diagnostic and/or forensic purposes. The subjects of the study were kept anonymous.

Open Access This article is licensed under a Creative Commons Attribution 4.0 International License, which permits use, sharing, adaptation, distribution and reproduction in any medium or format, as long as you give appropriate credit to the original author(s) and the source, provide a link to the Creative Commons licence, and indicate if changes were made. The images or other third party material in this article are included in the article's Creative Commons licence, unless indicated otherwise in a credit line to the material. If material is not included in the article's Creative Commons licence and your intended use is not permitted by statutory regulation or exceeds the permitted use, you will need to obtain permission directly from the copyright holder. To view a copy of this licence, visit http://creativecommons.org/licenses/by/4.0/.

\section{References}

1. Guthrie R, Susi A (1963) A simple phenylalanine method for detecting phenylketonuria in large populations of newborn infants. Pediatrics 32:338-343

2. Demirev PA (2013) Dried blood spots: analysis and applications. Anal Chem 85(2):779-789. https://doi.org/10.1021/ac303205m 
3. Wang L, Zeng Z, Emmons G (2010) Exploring the feasibility of using the DBS technique for metabolite radioprofiling. Bioanalysis 2:1365-1371. https://doi.org/10.4155/bio.10.100

4. Xie F, De Thaye E, Vermeulen A, Van Bocxlaer J, Colin P (2018) A dried blood spot assay for paclitaxel and its metabolites. J Pharm Biomed Anal 148:307-315. https://doi.org/10.1016/j. jpba.2017.10.007

5. Velghe S, De Troyer R, Stove C (2018) Dried blood spots in therapeutic drug monitoring and toxicology. Expert Opin Drug Metab Toxicol 14:1-3. https://doi.org/10.1080/17425255.2018.1414181

6. Chen G, Jirjees F, Al Bawab A, McElnay JC (2018) Quantification of amlodipine in dried blood spot samples by high performance liquid chromatography tandem mass spectrometry. J Chromatogr B Anal Technol Biomed Life Sci 1072:252-258. https://doi. org/10.1016/j.jchromb.2017.11.018

7. Capiau S, Veenhof H, Koster RA, Bergqvist Y, Boettcher M, Halmingh O, Keevil BG, Koch B, Linden R, Pistos C, Stolk L, Touw DJ, Stove C, Alffenaar JW (2019) Official international association for therapeutic drug monitoring and clinical toxicology guideline: development and validation of dried blood spotbased methods for therapeutic drug monitoring. Ther Drug Monit 41:409-430. https://doi.org/10.1097/FTD.0000000000000643

8. Xue KS, Cai W, Tang L, Wang J-S (2016) Aflatoxin B(1)-lysine adduct in dried blood spot samples of animals and humans. Food Chem Toxicol Assoc 98:210-219. https://doi.org/10.1016/j. fct.2016.11.002

9. Ross SA, Ahmed A, Palmer AL, Michaels MG, Sánchez PJ, Stewart A, Bernstein DI, Feja K, Fowler KB, Boppana SB, CMV and Hearing Multicenter Screening (CHIMES) Study Group (2017) Newborn dried blood spot polymerase chain reaction to identify infants with congenital cytomegalovirus-associated sensorineural hearing loss. J Pediatr. 184:57-61.e1. https://doi.org/10.1016/j. jpeds.2017.01.047

10. Bassaganyas L, Freedman G, Vaka D, Wan E, Lao R, Chen F, Kvale M, Currier RJ, Puck JM, Kwok P (2018) Whole exome and whole genome sequencing with dried blood spot DNA without whole genome amplification. Hum Mutat 39:167-171. https://doi. org/10.1002/humu.23356

11. Sadler Simões S, Castañera Ajenjo A, Dias MJ (2018) Dried blood spots combined to an UPLC-MS/MS method for the simultaneous determination of drugs of abuse in forensic toxicology. $\mathrm{J}$ Pharm Biomed Anal 147:634-644. https://doi.org/10.1016/j. jpba.2017.02.046
12. Moretti M, Visonà SD, Freni F, Tomaciello I, Vignali C, Groppi A, Tajana L, Osculati AMM, Morini L (2018) A liquid chromatography-tandem mass spectrometry method for the determination of cocaine and metabolites in blood and in dried blood spots collected from postmortem samples and evaluation of the stability over a 3-month period. Drug Test Anal 10:1430-1437. https://doi. org/10.1002/dta.2399

13. Moretti M, Freni F, Tomaciello I, Vignali C, Groppi A, Visonà SD, Tajana L, Osculati AMM, Morini L (2019) Determination of benzodiazepines in blood and in dried blood spots collected from post-mortem samples and evaluation of the stability over a three-month period. Drug Test Anal 11:1403-1411. https://doi. org/10.1002/dta.2653

14. Moretti M, Freni F, Valentini B, Vignali C, Groppi A, Visonà SD, Osculati AMM, Morini L (2019) Determination of antidepressants and antipsychotics in dried blood spots (DBSs) collected from post-mortem samples and evaluation of the stability over a three-month period. Molecules 24:3636. https://doi.org/10.3390/ molecules 24203636

15. Green H, Tillmar A, Pettersson G, Montelius K (2019) The use of FTA cards to acquire DNA profiles from postmortem cases. Int J Legal Med 133:1651-1657. https://doi.org/10.1007/s0041 4-019-02015-2

16. Lee H, Park Y, Jo J, In S, Park Y, Kim E, Pyo J, Choe S (2015) Analysis of benzodiazepines and their metabolites using DBS cards and LC-MS/MS. Forensic Sci Int 255:137-145. https:// doi.org/10.1016/j.forsciint.2015.07.004

17. Barfield M, Wheller R (2011) Use of dried plasma spots in the determination of pharmacokinetics in clinical studies: validation of a quantitative bioanalytical method. Anal Chem 83:118-124. https://doi.org/10.1021/ac102003t

18. Koster RA, Greijdanus B, Alffenaar JWC, Touw DJ (2014) Dried blood spot analysis of creatinine with LC-MS/MS in addition to immunosuppressants analysis. Anal Bioanal Chem 407:15851594. https://doi.org/10.1007/s00216-014-8415-2

Publisher's Note Springer Nature remains neutral with regard to jurisdictional claims in published maps and institutional affiliations. 